\title{
Comparison of ten rabbit lines of terminal bucks for growth, feed efficiency and carcass traits
}

\author{
Catherine LARZUL*, H. DE ROCHAMBEAU \\ Institut National de la Recherche Agronomique, Station d'Amélioration Génétique des Animaux, \\ BP 27, 31326 Castanet-Tolosan, France
}

(Received 27 November 2001; accepted 30 September 2004)

\begin{abstract}
Ten lines of rabbits differing by their growth pattern were compared based on their crossbred offspring performances. Paternal stocks were chosen from heavy and light lines from commercial or experimental populations. Offspring were measured on their daily gain, their feed efficiency and their carcass composition at 8,11 , and 14 weeks of age. It was evident that the growth pattern was transmitted to the offspring: animals sired from heavy lines were consistently heavier. The consequence on feed efficiency was as clear as for growth pattern: the offspring from heavy line bucks had a lower feed conversion ratio than those from light line bucks. Also, the same pattern was observed for adiposity. No pattern could be found for the muscle/bone ratio.
\end{abstract}

rabbit / growth rate / feed conversion / carcass

Résumé - Comparaison de 10 lignées de mâles terminaux pour les caractères de croissance, d'efficacité alimentaire et de composition corporelle chez le lapin. Dix lignées de lapins, différant par leur croissance, ont été comparées sur les résultats de leur descendance croisée. Les lignées paternelles ont été choisies dans des populations commerciales ou expérimentales sur la base de leur poids corporel. Les descendants ont été mesurés sur la croissance, l'efficacité alimentaire et la composition de la carcasse à 8,11 et 14 semaines d'âge. Il a été mis en évidence que les caractéristiques de la courbe de croissance se transmettaient à la descendance : les animaux les plus lourds sont issus des lignées les plus lourdes. Les conséquences sur l'efficacité alimentaire et l'adiposité des carcasses sont claires : les animaux les plus lourds sont les plus efficaces et les moins gras. En ce qui concerne la masse musculaire, aucune incidence de la croissance n'a pu être clairement mise en évidence.

lapin / croissance / efficacité alimentaire / carcasse

\footnotetext{
* Corresponding author: larzul@ dga.jouy.inra.fr Present Address: INRA-SGQA, Domaine de Vilvert, 78352 Jouy-en-Josas, France.
} 


\section{INTRODUCTION}

Rabbits commercially bred for meat production are mainly crossbred animals. They are usually produced by three-way crosses. Dam lines selected based on litter performances are crossed to produce the doe, mated to a buck from a line selected on weight gain. Crossbred does impart a heterosis effect on litter size $[4,23]$ and bucks contribute to growth complementarities.

In France, broilers are traditionally slaughtered at a live-weight of 2200-2400 g at about 70 days of age. This weight has remained constant for many years, but with the batch breeding system, slaughter tends to occur at a fixed age. Since genetic progress on post weaning growth rate has occurred [22], the slaughter age now tends to decrease steadily. It is known that age and live body weight at slaughter have major influences on carcass composition of meat-producing animals [8]. Consequently, the quality of the carcass and meat could change. The growth of the rabbit between birth or weaning and slaughter is well documented, but most studies deal with pure breeds and not with commercial lines of terminal bucks $[1,8,11,18$, 22]. Previous studies compared crossbred rabbits from several sire lines differing by their growth pattern at 77 days of age [12] or at different ages [13].

Therefore, the aim of the present study was to make a description of the growth pattern of 10 paternal lines, and to quantify at three ages the effect of the sire line used in crossbreeding with a standard French commercial doe, on the growth, food consumption and feed efficiency, as well as the carcass characteristics of market rabbits.

\section{MATERIALS AND METHODS}

\subsection{Animals}

\subsubsection{Breeding of the bucks and growth curve}

Bucks from 8 commercial lines and from 2 experimental lines were introduced just after their birth on an experimental farm (SELAP Station expérimentale lapin, INRA Toulouse). The commercial lines were provided by breeding companies. Line L9 was a control line (called 9077) involved in a selection experiment [23]. Line L10 (called 3889), originated from Dutch and Small Russian breeds. This slow growth line was previously selected for improving litter size. Breeding companies provided between 20 and 23 bucks per line and they were adopted by lactating does. They were weighed and weaned at 4 weeks of age.

Afterwards, the bucks were dispatched to another experimental farm (Le Magneraud, INRA Poitou Charentes). At 18 weeks of age, they were trained to produce semen. The frequency of semen collection was once a week for each buck. A sample of 11 bucks was chosen from all the bucks of each line on the basis of semen production. The bucks were weighed every 3 weeks from 8 to 59 weeks of age.

\subsubsection{Breeding of does}

One hundred and eighty does were introduced to Le Magneraud experimental farm just after weaning. They were born at the SELAP experimental farm. We crossed an A2066 buck with an A1077 doe to produce all 0067 females. This type of doe is used by many French rabbit breeders.

Rabbits were reared in an isolated, heated and dynamically ventilated building. Bucks and does received artificial lighting 16 hours per day. Each rabbit had an individual wire cage $\left(85 \times 40 \times 32 \mathrm{~cm}^{3}\right)$. Broiler rabbits were raised in another building. All rabbits were fed ad libitum with the same commercial pellet. Every three weeks, one series of inseminations was carried out, inseminating one half of the does. Only the semen of bucks of three lines was used in one series. The semen was mixed within the line and a balanced distribution of the semen of the three lines was attempted. In total, 14 series of inseminations were made between September 1994 and June 1995. From one series to 
another, 3 different buck lines were used in order to randomise the series effect.

\subsubsection{Breeding of broilers and growth traits}

About 550 young rabbits per series were weaned, weighed (BW) and tattooed at 35 days of age. They were housed in collective wire cages of six rabbits and the quantity of feed distributed in each cage was weighed. For all the abbreviated trait names, the subscript indicates age at measurement. For each series, at 8 weeks of age, 39 does (13 per male line) were weighed $\left(\mathrm{BW}_{8}\right)$ and slaughtered. At 11 weeks of age, 39 does (13 per male line $)$ were weighed $\left(\mathrm{BW}_{11}\right)$ and slaughtered, and 39 other does were transferred into individual wire cages to be weighed $\left(\mathrm{BW}_{14}\right)$ and slaughtered at 14 weeks of age. All the remaining broilers were sold at 11 weeks of age. The remaining feed was weighed for each cage. Therefore, daily gain from weaning to 8 weeks $\left(\mathrm{DG}_{\mathrm{w}-8}\right), 11$ weeks $\left(\mathrm{DG}_{\mathrm{w}-11}\right)$ and 14 weeks $\left(\mathrm{DG}_{\mathrm{w}-14}\right)$ was computed from these samples. Feed conversion ratio was computed for the same periods: weaning to 8 weeks, weaning to 11 weeks and weaning to 14 weeks, respectively $\mathrm{FCR}_{8}$, $\mathrm{FCR}_{11}$ and $\mathrm{FCR}_{14}$. In total, 16 slaughterings were realised between December 1994 and November 1995.

\subsection{Carcass traits}

A study of carcasses was made according to the recommendations made by Blasco and Ouhayoun [3]. Various measurements were taken after slaughtering: cold carcass weight $(\mathrm{CW})$, commercial carcass weight, perirenal fat weight $(\mathrm{PF})$, scapular fat weight (SF), fore part weight, intermediate part weight (Back), hind part weight (Hind), fresh hind leg weight, cooked hind leg weight, and hind leg bone weight (bone). Commercial carcass weight was also expressed as a percentage of live body weight, and perirenal fat weight, scapular fat weight, intermediate part weight and hind part weight as a percentage of the commercial carcass weight.
Muscle/bone ratio (M/B) was calculated as (fresh hind leg weight - hind leg bone weight) / hind leg bone weight. Cooking losses (CL) were calculated as the ratio (fresh hind leg weight - cooked hind leg weight) / fresh hind leg weight.

\subsection{Statistical analysis}

First, growth curves for bucks were fitted following the Gompertz model as described by Laird [9] since the Gompertz curve is appropriate to describe rabbit growth (Blasco and Gomez [2]).

$$
\mathrm{BW}_{\mathrm{t}}=\mathrm{BW}_{0} \exp (\mathrm{L} / \mathrm{K})(1-\exp (-\mathrm{Kt}))
$$

where $\mathrm{BW}_{\mathrm{t}}$ is the weight at age $\mathrm{t}, \mathrm{BW}_{0}$ is the weight at birth, $L$ is the initial specific growth rate, and $\mathrm{K}$ is the maturation rate. The coordinates of the point of inflexion, for which the growth rate is maximum, are $\mathrm{T}_{\mathrm{i}}$ and $\mathrm{BW}_{\mathrm{i}}$ with

$$
\begin{aligned}
T_{i}=\frac{1}{K} \ln \left|\frac{L}{K}\right| & \text {, and } \\
\mathrm{BW}_{\mathrm{i}} & =\mathrm{BW}_{0} \exp ((\mathrm{L} / \mathrm{K})-1) .
\end{aligned}
$$

First, the parameters of the Gompertz curve were estimated by line using the NLIN procedure of SAS software. As suggested by Pasternak and Shalev [17], the observations were weighted by the inverse of the variance of the weight. Only approximate tests are available to estimate the differences between lines for a non-linear regression. The differences between lines for the Gompertz curve parameters were tested using the SAS NLMIXED procedure, considering a normal distribution of the parameters. Even though the animals were weighed until 59 weeks of age, only weights between birth and 30 weeks of age were used in the analysis as suggested by Blasco and Gomez [2] in order to fit the first part of the curve.

The influence of the factors on the offspring traits was tested by an analysis of variance of fixed effects. The factors considered for growth traits were line and series 


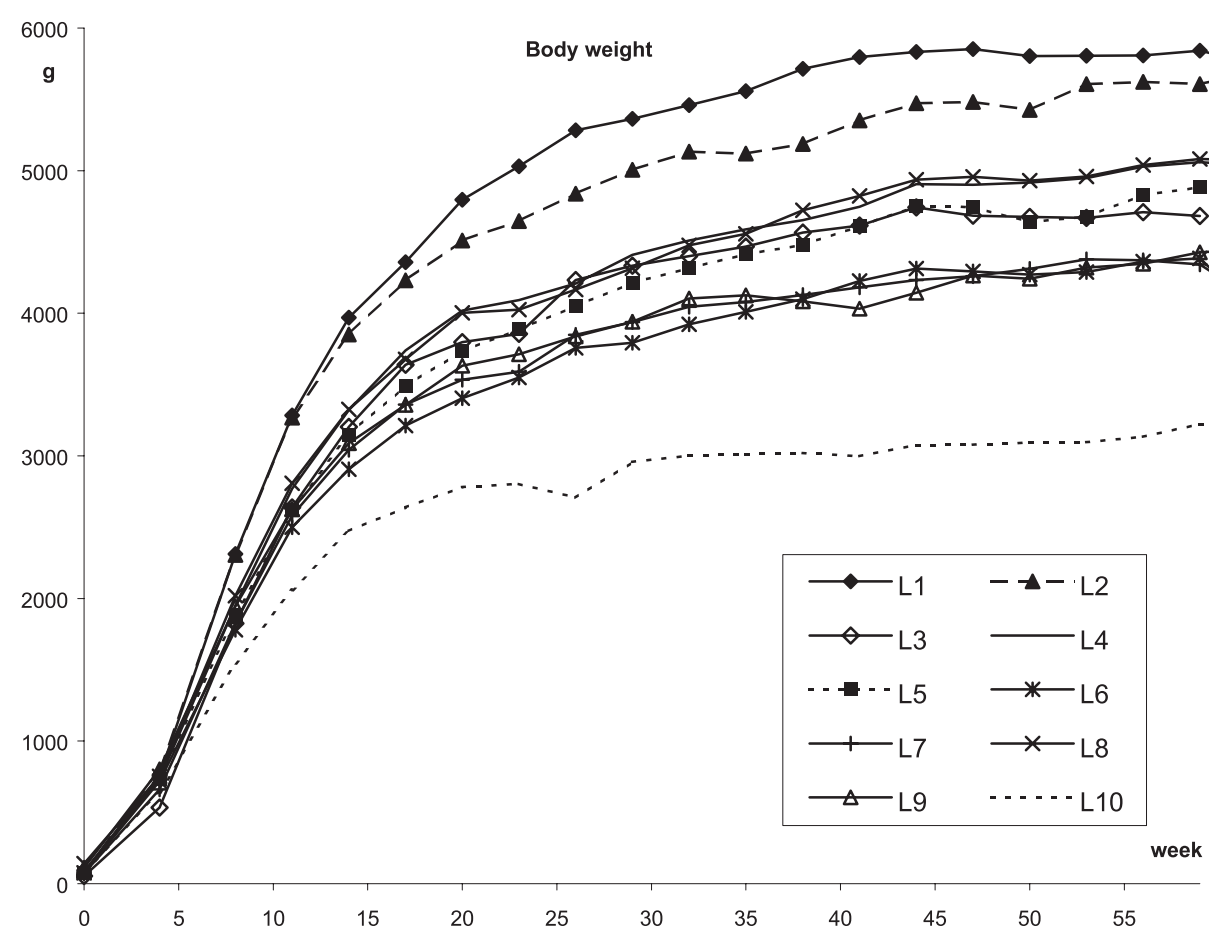

Figure 1. Evolution of body weight for the 10 buck lines.

of insemination, with these traits being analysed separately for the different ages. The same model was used to estimate the effects of the factors by a least squares approach. Concerning the carcass traits, the model used to test the significance of the factors by an analysis of variance included age, line, their interaction and the batch of slaughter as fixed effects. Another analysis was made within age to estimate the effect of the line and batch of slaughter using least squares. SAS was the software used to carry out all analyses.

The influence of different factors on the carcass traits was measured by fixed effect variance analysis. There were two fixed effects for the growth traits: series of weaning and bucks line. For slaughter traits, there were two fixed effects within age at slaughter: batch of slaughter and bucks line and the interaction between both effects. Data were analysed within age at slaughter $(8,11$ and 14 weeks). Data were computed with SAS version 8.2 software [25].

\section{RESULTS}

\subsection{Growth curves}

Mean growths per line are shown in Figure 1 . The difference between the curves taking into account weights until 30 weeks or 59 weeks of age is illustrated in Figure 2 for 3 lines. It was clear that including later weights in the analysis underestimated the first part of the curve. Parameters of the Gompertz curve for all lines are given in Table I. For body weight, the heaviest line was L1 and the lightest was L10. These results are in accordance with those previously observed for these lines. Other lines were more or less intermediate. Five groups 


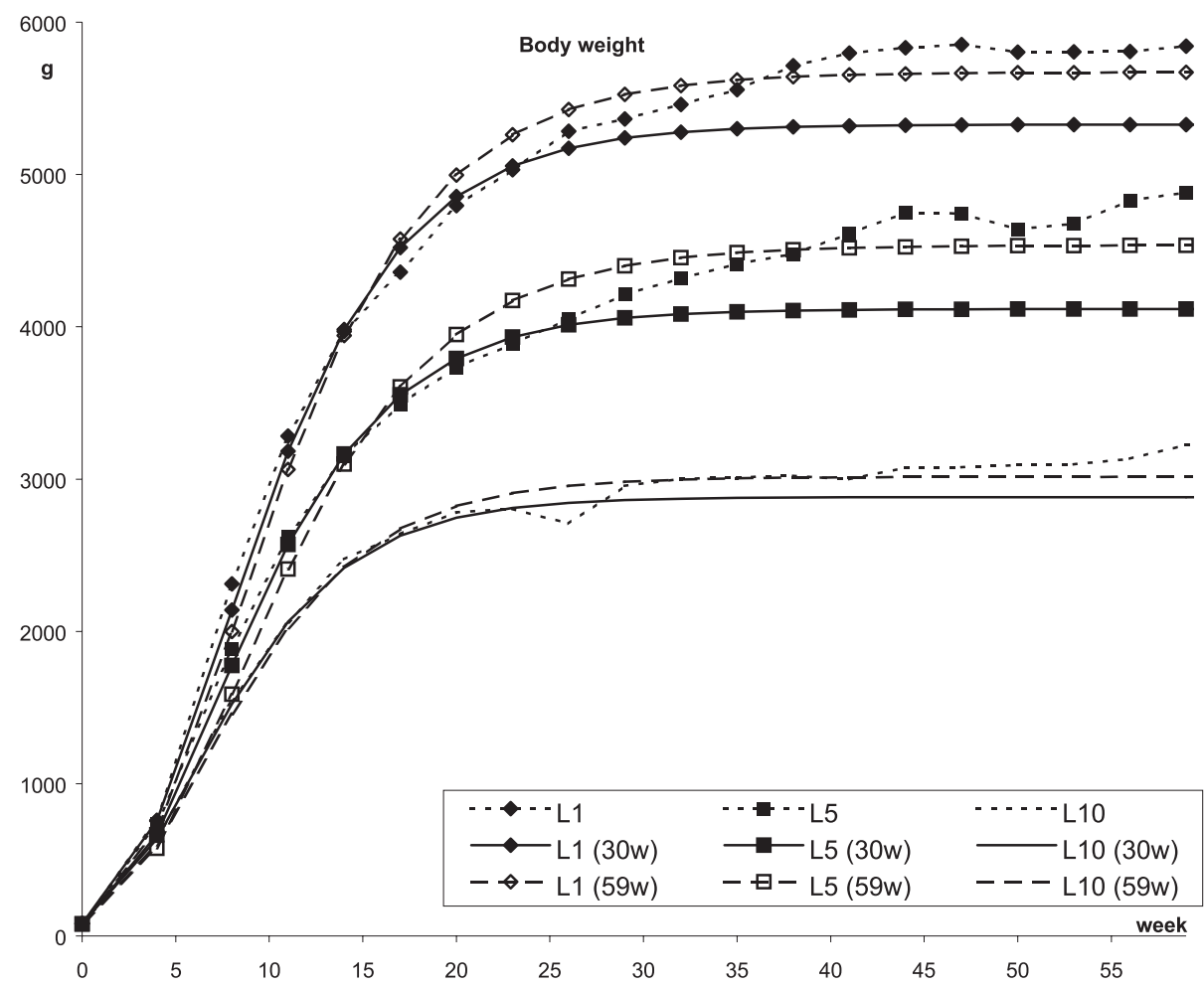

Figure 2. Evolution of measured body weight and predicted body weight by 2 Gompertz modelisations taking into account body weights until 30 or 59 weeks of age, for 3 buck lines.

Table I. Gompertz parameters of the growth curve for all buck lines (standard error in parentheses).

\begin{tabular}{lccccc}
\hline Line & $\mathrm{K}$ & $\mathrm{L}$ & $\mathrm{BW}_{0(\mathrm{~g})}$ & $\mathrm{T}_{\mathrm{i}(\text { days })}$ & $\mathrm{BW}_{\mathrm{i}(\mathrm{kg})}$ \\
\hline L1 & $0.0272(0.0007)^{\mathrm{ab}}$ & $0.114(0.007)^{\mathrm{bc}}$ & $81.4(13.3)^{\mathrm{ab}}$ & $52.6(0.7)^{\mathrm{d}}$ & $1.96(0.01)^{\mathrm{h}}$ \\
L2 & $0.0292(0.0009)^{\mathrm{bc}}$ & $0.120(0.009)^{\mathrm{bc}}$ & $80.9(15.3)^{\mathrm{ab}}$ & $48.4(0.7)^{\mathrm{c}}$ & $1.80(0.01)^{\mathrm{g}}$ \\
L3 & $0.0285(0.0010)^{\mathrm{abc}}$ & $0.127(0.011)^{\mathrm{c}}$ & $49.8(12.0)^{\mathrm{a}}$ & $52.3(0.8)^{\mathrm{d}}$ & $1.55(0.01)^{\mathrm{de}}$ \\
L4 & $0.0276(0.0009)^{\mathrm{abc}}$ & $0.109(0.008)^{\mathrm{abc}}$ & $84.7(16.2)^{\mathrm{abc}}$ & $49.7(0.8)^{\mathrm{c}}$ & $1.60(0.01)^{\mathrm{f}}$ \\
L5 & $0.0277(0.0009)^{\mathrm{abc}}$ & $0.109(0.008)^{\mathrm{abc}}$ & $78.8(14.9)^{\mathrm{ab}}$ & $49.7(0.8)^{\mathrm{c}}$ & $1.51(0.01)^{\mathrm{d}}$ \\
L6 & $0.0285(0.0011)^{\mathrm{abc}}$ & $0.111(0.010)^{\mathrm{abc}}$ & $76.3(16.2)^{\mathrm{ab}}$ & $47.7(0.9)^{\mathrm{bc}}$ & $1.38(0.01)^{\mathrm{b}}$ \\
L7 & $0.0290(0.0011)^{\mathrm{abc}}$ & $0.115(0.010)^{\mathrm{abc}}$ & $72.5(15.9)^{\mathrm{ab}}$ & $47.5(0.8)^{\mathrm{bc}}$ & $1.41(0.01)^{\mathrm{bc}}$ \\
L8 & $0.0263(0.0009)^{\mathrm{a}}$ & $0.091(0.008)^{\mathrm{a}}$ & $135.4(23.2)^{\mathrm{c}}$ & $47.2(0.8)^{\mathrm{bc}}$ & $1.59(0.01)^{\mathrm{ef}}$ \\
L9 & $0.0278(0.0010)^{\mathrm{abc}}$ & $0.099(0.008)^{\mathrm{ab}}$ & $111.7(19.6)^{\mathrm{bc}}$ & $45.3(0.8)^{\mathrm{b}}$ & $1.43(0.01)^{\mathrm{c}}$ \\
L10 & $0.0307(0.0015)^{\mathrm{c}}$ & $0.110(0.013)^{\mathrm{abc}}$ & $81.7(22.4)^{\mathrm{abc}}$ & $41.3(1.1)^{\mathrm{a}}$ & $1.06(0.01)^{\mathrm{a}}$ \\
\hline
\end{tabular}

$\mathrm{K}$ : maturation rate, $\mathrm{L}$ : initial specific growth rate, $\mathrm{BW}_{0}$ : weight at birth, $T_{i}=\frac{1}{K} \ln \left|\frac{L}{K}\right|$, and $\mathrm{BW}_{\mathrm{i}}=\mathrm{BW}_{0}$ $\exp ((\mathrm{L} / \mathrm{K})-1)$; values with the same letter are not significantly different at $P=0.05$. 
Table II. Level of significance of fixed effects for carcass traits.

\begin{tabular}{lcccc}
\hline Trait & Line & Batch & Age & Line $\times$ age \\
\hline BW & $* * *$ & $* * *$ & $* * *$ & $* * *$ \\
CW & $* * *$ & $* * *$ & $* * *$ & $* * *$ \\
Back & $* * *$ & $* * *$ & $* * *$ & $* * *$ \\
Hind & $* * *$ & $* * *$ & $* * *$ & $* * *$ \\
PF & $* * *$ & $* * *$ & $* * *$ & $* * *$ \\
SF & $* * *$ & $* * *$ & $* * *$ & $* * *$ \\
Bone & $* * *$ & $* * *$ & $* * *$ & $* * *$ \\
CW\% & $* * *$ & $* * *$ & $* * *$ & $*$ \\
Back\% & $* * *$ & $* * *$ & $* * *$ & NS \\
Hind\% & $* * *$ & $* * *$ & $* * *$ & $*$ \\
PF\% & $* * *$ & $* * *$ & $* * *$ & $* * *$ \\
SF\% & $* * *$ & $* * *$ & $* * *$ & $* *$ \\
M/B & $* * *$ & $* * *$ & $* * *$ & NS \\
CL\% & $*$ & $* * *$ & $* * *$ & $* *$ \\
\hline
\end{tabular}

BW: body weight, CW: carcass weight, Back: weight of the back part of the carcass, Hind: weight of the hind part of the carcass, PF: perirenal fat weight, SF: scapular fat weight, Bone: hind bone weight, M/B: hind muscle/bone ratio, CL: cooking losses; NS: not significant; $*$ : $P<0.05$; **: $P<$ $0.01 ; * * *: P<0.001$.

could be made up on the basis of age and weight at the point of inflexion: a heavy late-developing line (L1) and a heavy intermediate-developing line (L2), a medium late-developing line (L3) and medium intermediate-developing lines (L4, L5, L8, L7, L9, L6), and a very light precocious line (L10). In the medium intermediate group, the L8 line was characterised by a high body weight at birth in association with a low initial growth (L-value). The inflexion point was situated between 41.3 days of age for the most precocious and 52.6 days of age for the latest. The adult weights, given by $\mathrm{BW}_{\mathrm{i}} \mathrm{e}$, ranged from $2.9 \mathrm{~kg}$ for the lightest (L10) to $5.2 \mathrm{~kg}$ for the heaviest (L1).

\subsection{Broiler traits}

All broiler measurements were significantly affected by line and age (Tab. II). A significant interaction between age and line was found for all weights and percentages, except for the back part percentage and muscle/bone ratio for which this interaction was not found to be significant.

Weight and growth rates of broilers are given in Table III. At 11 weeks, offspring from the heavy group lines were heavier than those from the medium groups, which were heavier than those from the light line. The pattern was more complex between the medium groups. The differences increased with age. The weight difference between the extreme values was less than one standard deviation at weaning and more than two standard deviations at 14 weeks of age. Daily gain decreased with age between 8 and 11 weeks of age, and remained constant beyond this age.

Feed conversion ratios at the ages of 8 , 11 and 14 weeks for all lines are given in Table III. When comparing the differences between all lines at different ages, it appeared that the hierarchy between lines was not linked to paternal growth precocity. The lines with the lowest (L10, L9) or a high (L3) age at the inflexion point gave rabbits with similar feed conversion ratios, at least at 8 weeks of age. At 11 and 14 weeks of age, the L10 line rabbits had a significantly poorer feed conversion ratio. The $\mathrm{L} 1 \mathrm{rabbits}$ had the best conversion ratio at 11 and 14 weeks, but were not significantly different from the L2 rabbits at the latter age. These lines were the heaviest ones in our study, whereas the L10 was the lightest. It could only be concluded that heavy lines had a significantly better feed conversion ratio than the light line, with the mediumweight group being intermediate.

Least Squares Means and standard errors of all carcass traits were given in Table IV for weights and in Table $\mathrm{V}$ for percentages. We observed an increase in all weights from 8 to 14 weeks of age. In all lines, a large increase in carcass/live weight ratio was observed between 8 and 11 weeks of age, whereas the increase between 11 and 14 weeks was very limited or non-existent. There was no clear 
Table III. Least Square Means for growth traits of the offspring of all buck lines at 8, 11 and 14 weeks of age, and standard deviation (sd).

\begin{tabular}{lccccccccccc}
\hline Trait & L1 & L2 & L3 & L4 & L5 & L6 & L7 & L8 & L9 & L10 & $s d$ \\
\hline $\mathrm{BW}_{\mathrm{w}}$ & $978^{\mathrm{d}}$ & $933^{\mathrm{bc}}$ & $927^{\mathrm{bc}}$ & $932^{\mathrm{bc}}$ & $898^{\mathrm{a}}$ & $910^{\mathrm{ab}}$ & $898^{\mathrm{a}}$ & $907^{\mathrm{ab}}$ & $937^{\mathrm{c}}$ & $897^{\mathrm{a}}$ & 105 \\
$\mathrm{BW}_{8}$ & $1934^{\mathrm{f}}$ & $1861^{\mathrm{ef}}$ & $1800^{\mathrm{de}}$ & $1784^{\mathrm{cd}}$ & $1730^{\mathrm{abcd}}$ & $1686^{\mathrm{ab}}$ & $1744^{\mathrm{bcd}}$ & $1784^{\mathrm{cd}}$ & $1725^{\mathrm{abc}}$ & $1682^{\mathrm{a}}$ & 165 \\
$\mathrm{BW}_{11}$ & $2770^{\mathrm{f}}$ & $2691^{\mathrm{f}}$ & $2503^{\mathrm{cde}}$ & $2550^{\mathrm{e}}$ & $2423^{\mathrm{bc}}$ & $2354^{\mathrm{ab}}$ & $2454^{\mathrm{cd}}$ & $2534^{\mathrm{de}}$ & $2481^{\mathrm{cde}}$ & $2337^{\mathrm{a}}$ & 201 \\
$\mathrm{BW}_{14}$ & $3700^{\mathrm{e}}$ & $3513^{\mathrm{d}}$ & $3235^{\mathrm{c}}$ & $3344^{\mathrm{c}}$ & $3167^{\mathrm{abc}}$ & $3113^{\mathrm{ab}}$ & $3223^{\mathrm{bc}}$ & $3278^{\mathrm{c}}$ & $3264^{\mathrm{c}}$ & $3089^{\mathrm{a}}$ & 273 \\
$\mathrm{DG}_{\mathrm{w}-8}$ & $45.4^{\mathrm{f}}$ & $43.4^{\mathrm{ef}}$ & $40.5^{\mathrm{cd}}$ & $40.4^{\mathrm{cd}}$ & $39.2^{\mathrm{bc}}$ & $36.9^{\mathrm{a}}$ & $40.8^{\mathrm{cd}}$ & $41.9^{\mathrm{de}}$ & $38.7^{\mathrm{abc}}$ & $37.6^{\mathrm{ab}}$ & 5.0 \\
$\mathrm{DG}_{\mathrm{w}-11}$ & $42.8^{\mathrm{d}}$ & $42.0^{\mathrm{d}}$ & $37.8^{\mathrm{bc}}$ & $38.6^{\mathrm{c}}$ & $36.6^{\mathrm{b}}$ & $34.5^{\mathrm{a}}$ & $36.8^{\mathrm{b}}$ & $38.7^{\mathrm{c}}$ & $36.6^{\mathrm{b}}$ & $34.8^{\mathrm{a}}$ & 3.5 \\
$\mathrm{DG}_{\mathrm{w}-14}$ & $43.1^{\mathrm{f}}$ & $41.1^{\mathrm{e}}$ & $36.9^{\mathrm{cd}}$ & $38.3^{\mathrm{d}}$ & $35.9^{\mathrm{bc}}$ & $34.9^{\mathrm{ab}}$ & $36.8^{\mathrm{cd}}$ & $37.6^{\mathrm{cd}}$ & $36.6^{\mathrm{c}}$ & $34.4^{\mathrm{a}}$ & 3.5 \\
$\mathrm{FCR}_{8}$ & $2.54^{\mathrm{a}}$ & $2.63^{\mathrm{abc}}$ & $2.73^{\mathrm{cde}}$ & $2.78^{\mathrm{e}}$ & $2.66^{\mathrm{bcd}}$ & $2.75^{\mathrm{de}}$ & $2.64^{\mathrm{abcd}}$ & $2.59^{\mathrm{ab}}$ & $2.82^{\mathrm{e}}$ & $2.83^{\mathrm{e}}$ & 0.12 \\
$\mathrm{FCR}_{11}$ & $3.27^{\mathrm{a}}$ & $3.40^{\mathrm{bc}}$ & $3.46^{\mathrm{de}}$ & $3.43^{\mathrm{cd}}$ & $3.40^{\mathrm{bc}}$ & $3.53^{\mathrm{f}}$ & $3.44^{\mathrm{d}}$ & $3.39^{\mathrm{b}}$ & $3.49^{\mathrm{ef}}$ & $3.72^{\mathrm{g}}$ & 0.15 \\
$\mathrm{FCR}_{14}$ & $3.70^{\mathrm{a}}$ & $3.78^{\mathrm{ab}}$ & $3.97^{\mathrm{cd}}$ & $3.94^{\mathrm{d}}$ & $3.98^{\mathrm{cd}}$ & $4.07^{\mathrm{d}}$ & $3.97^{\mathrm{cd}}$ & $3.87^{\mathrm{bc}}$ & $3.96^{\mathrm{cd}}$ & $4.33^{\mathrm{e}}$ & 0.13 \\
\hline
\end{tabular}

$\mathrm{BW}$ : body weight in $\mathrm{kg}$, DG: daily gain in $\mathrm{g} \cdot \mathrm{d}^{-1}$, FCR: feed conversion ratio; subscript gives the age at the measurement $\mathrm{w}=$ weaning, $8=8$ weeks, $11=11$ weeks; $14=14$ weeks; within a row, values with the same letter are not significantly different at $P=0.05$.

Table IV. Least Square Means for carcass traits of the offspring of all buck lines at 8, 11 and 14 weeks of age, and standard deviation (sd).

\begin{tabular}{|c|c|c|c|c|c|c|c|c|c|c|c|}
\hline Trait & L1 & L2 & L3 & L4 & L5 & L6 & L7 & L8 & L9 & L10 & $s d$ \\
\hline $\mathrm{CW}_{8}$ & $1031^{d}$ & $998^{\mathrm{cd}}$ & $980^{c}$ & $955^{\mathrm{bc}}$ & $911^{\mathrm{abc}}$ & $916^{\mathrm{ab}}$ & $918^{\mathrm{ab}}$ & $951^{\mathrm{dc}}$ & $924^{\mathrm{ab}}$ & $901^{\mathrm{a}}$ & 108 \\
\hline $\mathrm{CW}_{11}$ & $1592^{\mathrm{e}}$ & $1546^{\mathrm{e}}$ & $1427^{\mathrm{cd}}$ & $1465^{\mathrm{d}}$ & $1356^{\mathrm{ab}}$ & $1322^{\mathrm{a}}$ & $1383^{\mathrm{abc}}$ & $1427^{\mathrm{cd}}$ & $1406^{\mathrm{bcd}}$ & $1335^{\mathrm{a}}$ & 134 \\
\hline $\mathrm{CW}_{14}$ & $2130^{\mathrm{f}}$ & $2030^{\mathrm{e}}$ & $1634^{\mathrm{bc}}$ & $1917^{d}$ & $1701^{\mathrm{abc}}$ & $1754^{\mathrm{ab}}$ & $1826^{\mathrm{abc}}$ & $1867^{\mathrm{cd}}$ & $1866^{\mathrm{cd}}$ & $1793^{b}$ & 171 \\
\hline $\mathrm{Back}_{8}$ & $249^{c}$ & $240^{c}$ & $235^{\mathrm{bc}}$ & $227^{\mathrm{ab}}$ & $223^{\mathrm{ab}}$ & $215^{\mathrm{a}}$ & $217^{\mathrm{a}}$ & $222^{\mathrm{a}}$ & $222^{\mathrm{a}}$ & $219^{a}$ & 29 \\
\hline Back $_{11}$ & $419^{\mathrm{e}}$ & $408^{d}$ & $370^{\mathrm{c}}$ & $377^{c}$ & $355^{\mathrm{a}}$ & $351^{\mathrm{a}}$ & $356^{\mathrm{ab}}$ & $381^{\mathrm{c}}$ & $366^{\mathrm{bc}}$ & $345^{\mathrm{f}}$ & 42 \\
\hline Back $_{14}$ & 573 & 548 & $490^{\mathrm{bcd}}$ & $512^{\mathrm{d}}$ & $487^{b c}$ & $470^{\mathrm{ab}}$ & $474^{\mathrm{abc}}$ & $495^{\mathrm{cd}}$ & $499^{\mathrm{cd}}$ & $466^{\mathrm{a}}$ & 52 \\
\hline $\mathrm{Hind}_{8}$ & $311^{\mathrm{e}}$ & $605^{\mathrm{e}}$ & $298^{\mathrm{de}}$ & $291^{\mathrm{cd}}$ & $283^{b c}$ & $275^{\mathrm{ab}}$ & $278^{a b c}$ & $285^{\mathrm{bcd}}$ & $279^{a b c}$ & $269^{\mathrm{a}}$ & 32 \\
\hline $\operatorname{Hind}_{11}$ & $499^{f}$ & $477^{\mathrm{e}}$ & $447^{\mathrm{d}}$ & $457^{\mathrm{d}}$ & $422^{b}$ & $418^{\mathrm{b}}$ & $426^{b c}$ & $451^{\mathrm{d}}$ & $440^{\mathrm{cd}}$ & $397^{\mathrm{a}}$ & 40 \\
\hline $\operatorname{Hind}_{14}$ & $637^{\mathrm{f}}$ & $599^{\mathrm{e}}$ & $554^{\mathrm{c}}$ & $583^{\mathrm{de}}$ & $550^{\mathrm{bc}}$ & $530^{\mathrm{ab}}$ & $549^{\mathrm{bc}}$ & $561^{\mathrm{cd}}$ & $560^{\mathrm{cd}}$ & $518^{\mathrm{a}}$ & 50 \\
\hline $\mathrm{PF}_{8}$ & $12.8^{\mathrm{bc}}$ & $11.8^{\mathrm{abc}}$ & $13.3^{\mathrm{c}}$ & $12.2^{\mathrm{bc}}$ & $11.6^{\mathrm{ab}}$ & $12.0^{\mathrm{bc}}$ & $10.0^{\mathrm{a}}$ & $12.4^{\mathrm{bc}}$ & $11.8^{\mathrm{bc}}$ & $16.4^{\mathrm{d}}$ & 4.1 \\
\hline $\mathrm{PF}_{11}$ & $30.5^{\mathrm{bc}}$ & $32.9^{c}$ & $30.2^{\mathrm{bc}}$ & $30.1^{\mathrm{bc}}$ & $25.7^{\mathrm{a}}$ & $25.8^{\mathrm{a}}$ & $29.2^{\mathrm{abc}}$ & $25.7^{\mathrm{a}}$ & $27.4^{\mathrm{ab}}$ & $44.3^{\mathrm{d}}$ & 9.7 \\
\hline $\mathrm{PF}_{14}$ & $63.4^{\mathrm{c}}$ & $65.1^{\mathrm{c}}$ & $58.4^{\mathrm{abc}}$ & $61.8^{\mathrm{bc}}$ & $53.2^{\mathrm{a}}$ & $61.1^{\mathrm{abc}}$ & $59.8^{\mathrm{abc}}$ & $54.1^{\mathrm{ab}}$ & $62.1^{\mathrm{bc}}$ & $86.4^{\mathrm{d}}$ & 19.2 \\
\hline $\mathrm{SF}_{8}$ & $6.0^{\mathrm{e}}$ & $4.9^{\mathrm{abc}}$ & $5.2^{\mathrm{bcde}}$ & $5.1^{\mathrm{bcd}}$ & $4.3^{\mathrm{a}}$ & $4.9^{\mathrm{abc}}$ & $4.5^{\mathrm{ab}}$ & $5.5^{\mathrm{cde}}$ & $5.4^{\text {cde }}$ & $5.8^{\mathrm{de}}$ & 1.8 \\
\hline $\mathrm{SF}_{11}$ & $9.6^{\mathrm{cd}}$ & $10.3^{\text {cde }}$ & $8.9^{\mathrm{bc}}$ & $9.2^{\mathrm{cd}}$ & $7.5^{\mathrm{a}}$ & $7.7^{\mathrm{ab}}$ & $9.6^{\mathrm{cd}}$ & $9.7^{\text {cde }}$ & $10.4^{\mathrm{de}}$ & $11.4^{\mathrm{e}}$ & 3.2 \\
\hline $\mathrm{SF}_{14}$ & $19.5^{\mathrm{e}}$ & $18.3^{\mathrm{de}}$ & $13.5^{\mathrm{ab}}$ & $16.6^{\mathrm{cd}}$ & $12.9^{\mathrm{a}}$ & $15.3^{\mathrm{bc}}$ & $15.0^{\mathrm{abc}}$ & $15.5^{\mathrm{bc}}$ & $16.8^{\mathrm{cd}}$ & $17.8^{\mathrm{de}}$ & 4.8 \\
\hline Bone $_{8}$ & $21.8^{\mathrm{f}}$ & $20.9^{e f}$ & $20.1^{\mathrm{cde}}$ & $20.5^{\mathrm{de}}$ & $19.2^{\mathrm{b}}$ & $19.2^{\mathrm{ab}}$ & $20.2^{\text {cde }}$ & $19.5^{\mathrm{bc}}$ & $19.8^{\mathrm{bcd}}$ & $18.4^{\mathrm{a}}$ & 2.0 \\
\hline Bone $_{11}$ & $28.5^{\mathrm{d}}$ & $27.9^{\mathrm{d}}$ & $25.7^{b c}$ & $26.4^{\mathrm{c}}$ & $25.0^{\mathrm{b}}$ & $24.9^{\mathrm{b}}$ & $26.3^{c}$ & $26.3^{c}$ & $26.1^{\mathrm{c}}$ & $23.3^{\mathrm{a}}$ & 2.2 \\
\hline Bone $_{14}$ & $32.9^{f}$ & $30.9^{\mathrm{e}}$ & $28.7^{\mathrm{abc}}$ & $30.1^{\mathrm{de}}$ & $29.1^{\mathrm{bcd}}$ & $28.1^{\mathrm{ab}}$ & $30.2^{\mathrm{de}}$ & $30.1^{\mathrm{de}}$ & $29.4^{\mathrm{cd}}$ & $27.7^{\mathrm{a}}$ & 2.8 \\
\hline
\end{tabular}

For abbreviations see Table II; within a row, values with the same letter are not significantly different at $P=0.05$. 
Table V. Least Square Means for carcass traits, in percentage of body weight or carcass weight, of the offspring of all buck lines at 8,11 and 14 weeks of age, and standard deviation (sd).

\begin{tabular}{|c|c|c|c|c|c|c|c|c|c|c|c|}
\hline Trait & L1 & L2 & L3 & L4 & L5 & L6 & L7 & L8 & L9 & L10 & $s d$ \\
\hline $\mathrm{W}_{8}$ & & $53.6^{\mathrm{ab}}$ & $54.4^{\mathrm{b}}$ & 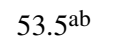 & $52.7^{\mathrm{a}}$ & $54.5^{\mathrm{b}}$ & $52.6^{\mathrm{a}}$ & $53.1^{\mathrm{ab}}$ & $53.4^{\mathrm{ab}}$ & $53.6^{\mathrm{ab}}$ & 3.6 \\
\hline $\mathrm{W}_{11}$ & $57.4^{\mathrm{c}}$ & $57.4^{\mathrm{c}}$ & $56.9^{\mathrm{bc}}$ & $57.5^{\mathrm{c}}$ & $55.9^{\mathrm{a}}$ & $56.2^{\mathrm{ab}}$ & $56.3^{\mathrm{ab}}$ & $56.2^{\mathrm{ab}}$ & $56.6^{\mathrm{abc}}$ & $57.1^{b c}$ & 2.2 \\
\hline $\mathrm{CW}_{14}$ & $57.5^{\mathrm{cde}}$ & $57.7^{\mathrm{de}}$ & $56.7^{\mathrm{ab}}$ & $57.2^{\mathrm{bcd}}$ & $56.8^{\mathrm{ab}}$ & $56.2^{\mathrm{a}}$ & $56.6^{\mathrm{ab}}$ & $56.9^{\mathrm{abc}}$ & $57.2^{\mathrm{bcd}}$ & $56.0^{\mathrm{a}}$ & 1.6 \\
\hline $\mathrm{ack}_{8}$ & $30.1^{\mathrm{abcd}}$ & $30.3^{\mathrm{bcd}}$ & $30.3^{\mathrm{abcd}}$ & $29.6^{\mathrm{a}}$ & $30.5^{\mathrm{d}}$ & $30.3^{\mathrm{cd}}$ & $29.8^{\mathrm{ab}}$ & $29.9^{\mathrm{abc}}$ & $30.0^{\mathrm{abcd}}$ & $30.3^{\mathrm{cd}}$ & 1.1 \\
\hline $\mathrm{ck}_{11}$ & $31.3^{\mathrm{abc}}$ & $31.7^{\mathrm{c}}$ & $31.5^{\mathrm{bc}}$ & $31.1^{\mathrm{ab}}$ & $31.7^{\mathrm{c}}$ & $31.5^{\mathrm{bc}}$ & $30.9^{\mathrm{a}}$ & $31.4^{\mathrm{bc}}$ & $31.2^{\mathrm{ab}}$ & $31.2^{\mathrm{ab}}$ & 1.0 \\
\hline Back $_{14}$ & $31.8^{\mathrm{bcd}}$ & $32.0^{\mathrm{cd}}$ & $31.7^{\mathrm{bcd}}$ & $31.5^{\mathrm{ab}}$ & $32.1^{\mathrm{d}}$ & $31.8^{\mathrm{bcd}}$ & $31.6^{\mathrm{bc}}$ & $31.6^{\mathrm{bc}}$ & $31.8^{\mathrm{bcd}}$ & $31.0^{\mathrm{a}}$ & 1.1 \\
\hline Hind $_{8}$ & $37.8^{\mathrm{ab}}$ & $38.5^{\mathrm{bc}}$ & $38.3^{\mathrm{bc}}$ & $38.6^{\mathrm{c}}$ & $38.8^{\mathrm{c}}$ & $38.4^{\mathrm{bc}}$ & $38.3^{\mathrm{bc}}$ & $20 \mathrm{pbc}$ & $38.0^{\mathrm{b}}$ & $37.3^{\mathrm{a}}$ & 1.4 \\
\hline $\operatorname{lnd}_{11}$ & $37.5^{\mathrm{c}}$ & $36.9^{\mathrm{b}}$ & $37.3^{\mathrm{bc}}$ & $37.8^{\mathrm{c}}$ & $37.4^{\mathrm{bc}}$ & $37.8^{c}$ & $37.2^{\mathrm{bc}}$ & $37.3^{b c}$ & $37.6^{\mathrm{c}}$ & & 1.3 \\
\hline $\operatorname{Hind}_{14}$ & $35.5^{\mathrm{bc}}$ & $34.9^{\mathrm{ab}}$ & $35.9^{\text {cde }}$ & $36.0^{\text {cde }}$ & $36.4^{\mathrm{e}}$ & $35.8^{\mathrm{cd}}$ & $36.0^{\text {cde }}$ & $36.3^{\mathrm{de}}$ & $35.7^{\mathrm{cd}}$ & $34.5^{\mathrm{a}}$ & 1.3 \\
\hline $\mathrm{PF}_{8}$ & $1.52^{\mathrm{abc}}$ & $1.44^{\mathrm{ab}}$ & $1.68^{\mathrm{c}}$ & $1.56^{\mathrm{bc}}$ & $1.55^{\mathrm{abc}}$ & $1.65^{\mathrm{bc}}$ & $1.35^{\mathrm{a}}$ & $1.61^{\mathrm{bc}}$ & $1.57^{\mathrm{bc}}$ & $2.28^{\mathrm{d}}$ & 0.45 \\
\hline $\mathrm{PF}_{11}$ & $2.18^{\mathrm{a}}$ & $2.59^{\mathrm{b}}$ & $2.54^{\mathrm{ab}}$ & $2.39^{\mathrm{ab}}$ & $2.29^{\mathrm{ab}}$ & $2.33^{\mathrm{ab}}$ & $2.49^{\mathrm{ab}}$ & & $2.32^{\mathrm{ab}}$ & & 0.85 \\
\hline $\mathrm{F}_{14}$ & $3.49^{\mathrm{ab}}$ & $3.91^{b c}$ & $3.71^{\mathrm{abc}}$ & $3.77^{\mathrm{abc}}$ & $3.46^{\mathrm{ab}}$ & $4.04^{\mathrm{c}}$ & $3.89^{\mathrm{bc}}$ & $3.37^{\mathrm{a}}$ & $3.93^{\mathrm{bc}}$ & $5.75^{\mathrm{d}}$ & 1.12 \\
\hline $\mathrm{SF}_{8}$ & $0.71^{\mathrm{cd}}$ & $0.62^{\mathrm{abc}}$ & $0.65^{\mathrm{abcd}}$ & $0.65^{\mathrm{abcd}}$ & $0.57^{\mathrm{a}}$ & $0.68^{\text {bcd }}$ & $0.61^{\mathrm{ab}}$ & $0.71^{\mathrm{d}}$ & $0.72^{\mathrm{d}}$ & $0.81^{\mathrm{e}}$ & 0.20 \\
\hline $\mathrm{SF}_{11}$ & $0.69^{\mathrm{a}}$ & $0.80^{\mathrm{abc}}$ & $0.75^{\mathrm{ab}}$ & $0.73^{\mathrm{ab}}$ & $0.66^{\mathrm{a}}$ & $0.69^{\mathrm{a}}$ & $0.83^{\mathrm{bc}}$ & $0.84^{\mathrm{bc}}$ & $0.87^{\mathrm{a}}$ & $1.03^{\mathrm{d}}$ & 0.26 \\
\hline $\mathrm{SF}_{14}$ & $1.07^{\mathrm{bc}}$ & $1.09^{\mathrm{bc}}$ & $0.85^{\mathrm{a}}$ & $1.01^{\mathrm{b}}$ & $0.84^{\mathrm{a}}$ & $1.01^{\mathrm{b}}$ & $0.98^{\mathrm{b}}$ & $0.97^{\mathrm{b}}$ & $1.06^{\mathrm{b}}$ & $1.18^{\mathrm{c}}$ & 0.27 \\
\hline $\mathrm{M} / \mathrm{B} 9$ & $5.44^{\mathrm{bc}}$ & $5.45^{\mathrm{bc}}$ & $5.58^{\mathrm{c}}$ & $5.38^{\mathrm{bc}}$ & $5.47^{\mathrm{bc}}$ & $5.39^{\mathrm{bc}}$ & $5.13^{\mathrm{a}}$ & $5.52^{\mathrm{bc}}$ & $5.32^{\mathrm{ab}}$ & $5.51^{b c}$ & 0.48 \\
\hline $\mathrm{M} / \mathrm{B}_{11}$ & $6.65^{\mathrm{c}}$ & $6.60^{\mathrm{bc}}$ & $6.62^{\mathrm{bc}}$ & $6.67^{\mathrm{c}}$ & $6.50^{\mathrm{bc}}$ & $6.42^{\mathrm{ab}}$ & $6.24^{\mathrm{a}}$ & $6.61^{b c}$ & $6.49^{\mathrm{bc}}$ & $6.55^{\mathrm{bc}}$ & 0.49 \\
\hline $\mathrm{M} / \mathrm{B}_{14}$ & $7.59^{\mathrm{d}}$ & $7.39^{\mathrm{bcd}}$ & $7.55^{\mathrm{cd}}$ & $7.57^{\mathrm{cd}}$ & $7.29^{\mathrm{b}}$ & $7.31^{b c}$ & $6.93^{\mathrm{a}}$ & $7.34^{\mathrm{bcd}}$ & $7.40^{\mathrm{bcd}}$ & $7.25^{\mathrm{b}}$ & 0.59 \\
\hline & $28.7^{\mathrm{ab}}$ & $28.4^{\mathrm{ab}}$ & $29^{\mathrm{b}}$ & $28.3^{\mathrm{a}}$ & $28.2^{\mathrm{a}}$ & $28.5^{\mathrm{ab}}$ & $28.2^{\mathrm{ab}}$ & $28.5^{\mathrm{ab}}$ & $28.3^{\mathrm{ab}}$ & $28.1^{\mathrm{a}}$ & 1.5 \\
\hline $\mathrm{CL}_{11}$ & $29.3^{\mathrm{ab}}$ & $29.4^{\mathrm{b}}$ & $28.9^{\mathrm{ab}}$ & $28.5^{\mathrm{a}}$ & & & $29.0^{\mathrm{ab}}$ & $28.8^{\mathrm{ab}}$ & $29.1^{\mathrm{ab}}$ & $28.6^{\mathrm{a}}$ & 1.7 \\
\hline $\mathrm{CL}_{14}$ & $28.6^{\mathrm{c}}$ & $27.7^{\mathrm{a}}$ & $28.6^{\mathrm{c}}$ & $28.6^{\mathrm{c}}$ & $28.4^{\mathrm{bc}}$ & $27.7^{\mathrm{ab}}$ & $28.9^{\mathrm{c}}$ & $28.2^{\mathrm{abc}}$ & $28.4^{\mathrm{c}}$ & $28.6^{\mathrm{c}}$ & 1.6 \\
\hline
\end{tabular}

For abbreviations see Table II; within a row, values with the same letter are not significantly different at $P=0.05$.

pattern for the carcass/live weight ratio between the five groups. At 11 weeks, line L1 and L10 were not significantly different. Line L4 had the highest ratio and line L5, from the same group had the lowest. In all lines, the back part percentage increased from 8 to 14 weeks, and the hind part percentage decreased from 8 weeks to 14 weeks. Depending on the line, the hind part percentage decrease was more important between 8 and 11 or between 11 and 14 weeks. For example, in the L1 line, the decrease was higher between 11 and 14 weeks; for L2 or L10, the decrease was continuous. The L10 always had the lowest hind part percentage, regardless of age.
For perirenal fat percentage, a major indicator of the adiposity of the carcass for it is the latest developing dissectible adipose tissue, the L10 line always had significantly higher values, regardless of age. For the other lines, the differences changed in relation with age. If we consider the heaviest lines: at 11 and 14 weeks of age, L1 tended to be in the low fat-percentage-value group and L2 tended to be in the high fat-percentage-value group. If we consider late lines (L3 and L1) and a more precocious line (L9) the difference was not significant. For the muscle/bone ratio, a major indicator of the total amount of meat in the carcass, no pattern could be found to explain the differences 
between the lines, except that the L7 line had the lowest muscle/bone ratio, regardless of age. For cooking losses, the differences between lines depend on the age and the order may vary. Nevertheless, for all lines cooking losses tended to be higher at 11 weeks of age than at other ages.

\section{DISCUSSION}

\subsection{Growth curves}

Growth curves were previously fitted for rabbits using 3 parameter curves $[1,2,7,10$, $11,19,20,24,26]$ or 4 parameter curves $[2$, 26]. It appears that the Gompertz function is appropriate enough to describe growth rate for a large variety of breeds. As suggested by Blasco and Gomez [2], the estimated adult weight was underestimated in comparison with the observed 59 week weight. After puberty, the increasing weight may have been due to fat deposition. In this case, growth curves never show a plateau and weight still increased during aging. In order to overcome this difficulty, weights over 30 weeks of age were not taken into account in the estimation of the Gompertz curve parameters.

The results obtained in this study were in agreement with previous values reported by Blasco and Gomez [3] for growth curve parameters, with a maximum growth within the 7 th week of age, and the inflexion point at 53 or 54 days of age. When comparing several lines for growth, the results are quite different. In a review, Lehmann [10] reports ages at the inflexion point for different line or crossbred rabbits varying from 44 to 63 days of age. It is generally believed that an increasing growth rate decreases precocity (at the age of slaughtering, the weight of the animals as a ratio of the adult weight decreases). In a recent study, a comparison of animals selected and not selected for growth rate showed a completely equal slope of the Gompertz curve [21]. In other words, the animals selected had the same growth curve as the control animals. In this hypothesis, selection for growth rate would lead to less mature animals only when slaughtering is fixed at a constant weight. If the animals are slaughtered at a constant age, their maturity would not be affected. Comparing animals selected on growth rate vs. animals selected on litter size, Blasco and Gomez [3] also found very similar results for growth curve parameters in both lines. Since we found some differences between the lines for growth curve parameters, it could be hypothesised that the genetic background more than the selection process in commercial lines has induced different growth patterns. Previously, Lehmann [11] with a modified Gompertz curve modelisation found some differences between New Zealand (46 or 49 days of age at the inflexion point) and Californian rabbits (41 days of age).

\subsection{Broiler traits}

The decrease in daily gains with age was in accordance with the growth pattern. As stated by Ouhayoun [15] and described in several studies including the present one, growth rate is maximum between 5 and 7 weeks of age. When considering posterior ages, it was expected that daily gains would decrease or at least remain constant.

Previous results have shown that with the increase of age, adiposity of the carcass increased as well as the muscle/bone ratio. Cantier et al. [5] estimated the allometry coefficients in the rabbit for several tissues. They found that the muscle tissue increased more rapidly than the body until the weight of $2.4 \mathrm{~kg}$. Thus, from this weight, the muscle/bone ratio was decreasing. Varewyck and Bouquet [27] also found that this ratio did not increase after 11 weeks of age (which corresponded to $2.4 \mathrm{~kg}$ ). In our study, the results were not in accordance with the results mentioned above. The increase of muscle/bone ratio was obvious for all lines from 8 weeks until 14 weeks of age. To explain such a discrepancy, one must consider that our breeding techniques and actual lines were very different from those 
used by Cantier et al. [5] or by Varewyck and Bouquet [27].

Ouhayoun [14] found a maternal effect: the differences in body weight between crossbred animals from several lines, differing in adult body weight, were only significant from 70 days of age and beyond. In the present study, the differences were significant at weaning age. The heaviest line crossbred animals showed a heavier weaning weight. For the other lines, differences were less significant, for example between animals L5 and L7 on the one hand and animals L10 on the other hand, whereas their adult body weights were completely different. It should be emphasised that the genetic differences between sire lines was divided by two in crossbred animals, which could also explain the low significant differences between all lines, except for the heaviest. But the rank given by the adult sire body weight was maintained in crossbred animals. When ordering the lines, for example on the basis of body weight at 11 weeks of age, we found the same order as given by the adult sire body weight, except for L5 animals, which appeared somewhat lighter than the expected adult weight. Thus, when differences between lines were expressed as standard deviation, the weight differential was enhanced at 14 weeks of age in comparison with weaning weight, which could be an indication of maternal effects countering the genetic differences.

In their study, Feki et al. [6], comparing rabbits from lines selected on growth rate or litter size, associated the differences of feed conversion ratio to growth rate improvement. In our study, it was clear that lines with the highest growth rate also had the best feed conversion ratio at 14 weeks of age. However, at 11 weeks of age, this conclusion was not supported by our data; rabbits from lines differing by their growth rate (L2, L5, L8) presented equal feed conversion ratios; and lines with similar growth rates (L3, L8) had significantly different feed conversion ratios. Heavy lines, how- ever, had a better feed conversion ratio than the light line.

Ouhayoun [16] reported that carcass composition in crossbred rabbits from different lines differing by their adult weight does not depend on maturity or growth rate: at the age of 11 weeks, animals with the higher degree of maturity were not necessarily the animals with the higher dressing out percentage or the higher muscle/bone ratio. Rabbits from the light adult weight line had the highest carcass adiposity. Our results were in agreement with these previous statements, which could be extended to 8 or 14 weeks of age. The rabbits from the L10 sire line, which had the lightest adult weight and the highest maturity degree, were also fattier, expressed in terms of absolute weight or in percentage of the carcass weight. But, the high fatness was not due to precocity. The L9 line, which was nearly as precocious as the L10 line, was not as fat. This could be explained by a combination between precocity and light adult weight, or by a completely different genetic background not in relation with the growth pattern.

\section{REFERENCES}

[1] Baron R., Vezinhet A., Cantier J., Allométrie de croissance chez le lapin. II. Thymus, Ann. Biol. Ann. Biochem. Biophys. 10 (1970) 535548.

[2] Blasco A., Gomez E., A note on growth curves of rabbit lines selected on growth rate or litter size, Anim. Prod. 57 (1993) 332-334.

[3] Blasco A., Ouhayoun J., Harmonization of criteria and terminology in rabbit meat research. Revised proposal, World Rabbit Sci. 4 (1996) 93-99.

[4] Brun J.M., Rouvier R., Evolution of genetic parameters of litter traits in crosses of two selected strains of rabbits: a synthesis, in: Proceeding of the 4th World Rabbit Science Association, Budapest, 1988, pp. 158-166.

[5] Cantier J., Vezinhet A., Rouvier R., Dauzier L., Allométrie de croissance chez le lapin (Oryctolagus cuniculus). 1/ Principaux organes et tissu, Ann. Biol. Ann. Biochem. Biophys. 9 (1969) 5-39. 
[6] Feki S., Baselga M., Blas E., Cervera C., Gomez E.A., Comparison of growth and feed efficiency among rabbit lines selected for different objectives, Livest. Prod. Sci. 45 (1996) 87-92.

[7] Fl'ak P., Growth of body weight in New Zealand White Rabbit, in: Proceeding of the 2nd International Colloquy "The rabbit as a model animal and breeding object”, 15-17 Sept 1982, Rostock, Wilhelm-Pieck-Universitat Rostock Sektion Tierproduktion, 1983, pp. 75-78.

[8] Gomez E.A., Baselga M., Rafel O., Ramon J., Comparison of carcass characteristics in five strains of meat rabbit selected on different traits, Livest. Prod. Sci. 55 (1998) 53-64.

[9] Laird A.K., Postnatal growth of birds and mammals, Growth 30 (1965) 349-363.

[10] Lehmann R., Zum postnatalen Wachsumsverlauf von Kaninchen, in: Proceeding of the 2 nd International Colloquy "The rabbit as a model animal and breeding object”, 22-23 March 1979, Rostock, Wilhelm-Pieck-Universitat Rostock Sektion Tierproduktion, 1980, pp. 27-36.

[11] Lehmann R., Untersuchungen zum Wachstumsverkauf von Kaninchen, in: Proceeding of the 2nd International Colloquy "The rabbit as a model animal and breeding object", 1517 Sept 1982, Rostock, Wilhelm-Pieck-Universitat Rostock Sektion Tierproduktion, 1983, pp. 67-74.

[12] Masoero G., Breeding and crossbreeding to improve growth rate, feed efficiency and carcass characters in rabbit meat production, in: Proceeding of the 2nd Congress of Genetic Applied to Livestock Production, Madrid, Vol. 6, 1982, pp. 499-509.

[13] Ouhayoun J., Étude comparative de races de lapins différant par le poids adulte; incidence du format paternel sur les composantes de la croissance des lapereaux issus de croisement terminal, Thèse, Montpellier, France, 1978.

[14] Ouhayoun J., Évolution comparée de la composition corporelle de lapins de trois types génétiques, au cours du développement postnatal, Reprod. Nutr. Dev. 20 (1980) 949-959.

[15] Ouhayoun J., La croissance et le développement du lapin de chair, Cuni-Sciences 1 (1983) 1-15.

[16] Ouhayoun J., La composition corporelle du lapin: facteurs de variation, INRA Prod. Anim. 2 (1989) 215-226.
[17] Pasternak H., Shalev B.A., The effect of feature of regression disturbance on the efficiency of fitting a growth curve, Growth Dev. Aging 58 (1994) 33-39.

[18] Pla M., Guerrero L., Guardia D., Oliver M.A., Blasco A., Carcass characteristics and meat quality of rabbit lines selected for different objectives: I. Between lines comparison, Livest Prod. Sci. 54 (1998) 115-123.

[19] Ptak E., Bieniek J., Jagusiak W., Comparison of growth curves of purebred and crossbred rabbits, in: Proceeding of the 5th World Congress of Genetic Applied to Livestock Production, Guelph, Canada, 7-12 August 1994, Vol. 19, pp. 201-204.

[20] Piles M. Blasco A., Pla M., The effect of selection for growth rate on carcass composition and meat characteristics of rabbits, Meat Sci. 54 (2000) 347-355.

[21] Piles M., Blasco A., Varona L., Pla M., Correlated response to selection on growth curves in rabbits selected for increasing growth rate, in: Proceeding of the 7th World Congress on Rabbit, Valencia, Spain, 4-7 July 2000, 651658.

[22] de Rochambeau H., [Genetics of meat-producer rabbit], INRA Prod. Anim. 2 (1989) 287-295.

[23] de Rochambeau H., La femelle parentale issue des souches expérimentales de l'INRA : évolutions génétiques et perspectives, in: Procee-

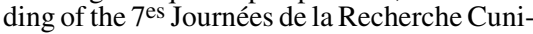
cole, Lyon, 13-14 mai 1998, pp. 3-14.

[24] Sager G., Mathematical formulations of rabbit weight growth after data from Templeton (1955), in: Proceeding of the 2nd International Colloquy "The rabbit as a model animal and breeding object”, 15-17 Sept 1982, Rostock, Wilhelm-Pieck-Universitat Rostock Sektion Tierproduktion, 1983, pp. 79-86.

[25] SAS, SAS Institute Inc., SAS OnlineDoc®, Version 8, Cary, NC, USA, 1999

[26] Seeland G., Rossler B., Roder B., Analysis of growth in rabbits of different breeds with selected growth function, Arch. Tierzucht 39 (1996) 533-544.

[27] Varewyck H., Bouquet Y., Relations entre la composition tissulaire de la carcasse de lapins de boucherie et celle des principaux morceaux, Ann. Zootech. 31 (1982) 257-268. 